\title{
Mammalian Models of Duchenne Muscular Dystrophy: Pathological Characteristics and Therapeutic Applications
}

\author{
Akinori Nakamura ${ }^{1}$ and Shin'ichi Takeda ${ }^{2}$ \\ ${ }^{1}$ Department of Medicine (Neurology and Rheumatology), School of Medicine Shinshu University, 3-1-1 Ahahi, \\ Matsumoto 390-8621, Japan \\ ${ }^{2}$ Department of Molecular Therapy, National Institute of Neuroscience, National Center of Neurology and Psychiatry, \\ 4-1-1 Ogawa-higashi, Kodaira, Tokyo 187-8502, Japan \\ Correspondence should be addressed to Akinori Nakamura, anakamu@shinshu-u.ac.jp
}

Received 14 October 2010; Accepted 19 December 2010

Academic Editor: Andrea Vecchione

Copyright (๑) 2011 A. Nakamura and S. Takeda. This is an open access article distributed under the Creative Commons Attribution License, which permits unrestricted use, distribution, and reproduction in any medium, provided the original work is properly cited.

\begin{abstract}
Duchenne muscular dystrophy (DMD) is a devastating X-linked muscle disorder characterized by muscle wasting which is caused by mutations in the DMD gene. The DMD gene encodes the sarcolemmal protein dystrophin, and loss of dystrophin causes muscle degeneration and necrosis. Thus far, therapies for this disorder are unavailable. However, various therapeutic trials based on gene therapy, exon skipping, cell therapy, read through therapy, or pharmaceutical agents have been conducted extensively. In the development of therapy as well as elucidation of pathogenesis in DMD, appropriate animal models are needed. Various animal models of DMD have been identified, and mammalian (murine, canine, and feline) models are indispensable for the examination of the mechanisms of pathogenesis and the development of therapies. Here, we review the pathological features of DMD and therapeutic applications, especially of exon skipping using antisense oligonucleotides and gene therapies using viral vectors in murine and canine models of DMD.
\end{abstract}

\section{Introduction}

Duchenne muscular dystrophy (DMD) is a lethal X-linked muscle disease characterized by progressive skeletal muscle atrophy and weakness [1]. Mutations in the causative gene $D M D$ result in loss of the cytoskeletal protein dystrophin. This is accompanied by a defect of dystrophin-glycoprotein complex (DGC) in the sarcolemma and leads to progressive muscle degeneration [2, 3]. The dystrophic-deficient skeletal muscle exhibits muscle fiber necrosis with invasion of inflammatory cells followed by muscle regeneration. The muscle is progressively replaced by fibrous or fatty tissue. Recent advances in molecular biology have identified murine, canine, and feline DMD animal models [4]. Efforts to develop various therapeutic approaches such as gene therapy, cell therapy, or pharmaceutical agents have been conducted using DMD animal models, although no radical and permanent therapy is available. In this review, we describe the pathological characteristics and availability of therapeutic applications with a focus on gene therapy in mammalian models of DMD.

\section{Duchenne Muscular Dystrophy}

2.1. Pathogenesis of DMD. Muscular dystrophies are inherited, and progressive muscle disorders are characterized by muscle fiber degeneration and necrosis. Duchenne muscular dystrophy (DMD) is the most severe and common X-linked disorder (1 in 3,500 male births) [1]. The onset of this disorder is recognized by observation of walking difficulties experienced by children between 2 and 5 years of age. The skeletal muscle degeneration is progressive, resulting in patients being wheelchair bound by the age of 13. DMD patients tend to die by the age of about 30 as a result of respiratory or cardiac failure [1].

DMD is caused by a mutation in the DMD gene, which is one of the largest in the human genome (approximately 2.5 million base pairs, encoding 79 exons). Full-length 
dystrophin mRNA is approximately $14 \mathrm{~kb}$ and is mainly expressed in skeletal, cardiac, and smooth muscles, as well as in the brain [2]. The dystrophin protein encoded by the $D M D$ gene is rod shaped and consists of 4 domains: the $\mathrm{N}$-terminal actin-binding domain, a rod-shaped domain composed of 24 spectrin-like rod repeats and 4 hinges, a cysteine-rich domain that interacts with dystroglycan and sarcoglycan complexes, and the C-terminal domain that interacts with the syntrophin complex and dystrobrevin [3]. Dystrophin is localized at the sarcolemma and forms a dystrophin-glycoprotein complex (DGC) with dystroglycan, sarcoglycan, and syntrophin/dystrobrevin complexes. These associations link the cytoskeletal protein actin to the basal lamina of muscle fibers [3]. DGC is thought to act as a membrane stabilizer during muscle contraction or a transducer of signals from the extracellular matrix to the muscle cytoplasm via intracellular signaling molecules. Loss of dystrophin leads to conditions under which the membrane becomes leaky as a result of mechanical or hypoosmotic stress. Consequently, $\mathrm{Ca}^{2+}$ permeability is increased and various $\mathrm{Ca}^{2+}$ dependent proteases such as calpain are activated under conditions of dystrophin deficiency. It has also been proposed that alteration of the expression or function of the plasma membrane proteins associated with dystrophin such as neuronal nitric oxide synthase (nNOS) and various ion channels are involved in the molecular mechanisms of muscle degeneration [5].

The DMD gene mutations include missense, nonsense, deletion, insertion, or duplication mutations. When a mutation of DMD disrupts the reading frame of amino acids (an out-of-frame mutation), the dystrophin defect results in the severe DMD phenotype. On the other hand, a mutations which maintains the reading frame (an in-frame mutation) tends to produce a truncated but functional dystrophin, which leads to the more benign phenotype known as Becker muscular dystrophy (BMD). About $90 \%$ of cases of the phenotypes of DMD and BMD can be explained by this frameshift theory [6]. There are two major hot spots for mutations around exons 3-7 and exons 45-55 in the DMD gene [6].

\subsection{Investigations of Gene Therapy for Treatment of DMD.} For the treatment of DMD, various experimental approaches such as gene therapy using viral vectors, exon-skipping therapy, stem cell transplantation, read-through therapy, and pharmacological agents have been extensively developed, but none of these have met with success in the clinic. Among these therapeutic strategies, exon skipping using antisense oligonucleotides (AOs) has been considered to be one of the most promising therapies for the restoration of dystrophin expression at the sarcolemma in dystrophin-deficient muscle. Exon skipping as treatment for DMD is developed based on the frame-shift theory. AOs are chemically-synthesized single-strand nucleic acids about 25 bases in length which are designed to recognize a specific sequence of the mRNA splicing pattern or of the binding protein. These agents can artificially change the translation of the nucleic acid. Among the various types of $\mathrm{AOs}$, the $2^{\prime}$-O-methyl phosphorothioate $\mathrm{AO}(2 \mathrm{OMeAO})$ and the phosphorodiamidate morpholino oligomers (PMO: morpholino) have high efficacy, low toxicity, and high stability $[7,8]$. Therefore, exon skipping strategies mediated by $2 \mathrm{OMeAO}$ and PMO have been extensively performed and clinical studies have been conducted.

Gene therapy using viral vectors has been extensively investigated. Adeno-associated virus (AAV) vectors are the most appropriate tools for viral vector gene therapy because they are nonpathogenic due to a replication defect and have low immunity with an effective ability to infect nondividing cells. This strategy, however, is limited with respect to the size of the inserted exogenous gene. The upper limit of $4.9 \mathrm{~kb}$ prevents the full-length $D M D$ cDNA $(14 \mathrm{~kb})$ from being successfully incorporated into the vector. Our group and other researchers have designed a shorter but functional microdystrophin, which is driven by the muscle-specific creatine kinase (MCK) promoter in combination with AAV vectors of various serotypes. The efficacy of this system has been examined $[9,10]$, and it has been found that the immune reaction is one of the critical issues in canine or primates. Other serotypes of AAV with a weaker immune reaction, such as AAV8, have recently been developed. These rAAVs are capable of providing powerful systemic delivery of the $D M D$ gene to muscles throughout the body, including the heart.

\section{Mammalian Models of DMD}

Animal models are needed for elucidation of the pathogenesis and assessment of efficacy and toxicity during the development of therapies. In DMD, various animal models have been identified and utilized. In this review, we introduce and discuss not only the pathological characteristics of mammalian (murine, feline, or canine) models of DMD but also recent advances in therapeutic applications using these models.

\section{Murine Model of DMD}

Various mouse models with mutations in the mouse $D M D$ gene have been identified: X-linked muscular dystrophy mouse $(m d x)$ [11] and 4 additional strains of $m d x$ mouse$m d x 2 \mathrm{cv}, m d x 3 \mathrm{cv}, m d x 4 \mathrm{cv}$, or $m d x 5 \mathrm{cv}$ mouse [12]. Moreover, the $D M D$ exon 52 knockout $(m d x 52)$ mouse [13] and the $m d x$ mouse with additional ablation of the dystrophin homologue utrophin $\left(m d x / u t r^{-/-}\right)$[14] was produced. Among these models, the $m d x$ mouse is the most commonly used model. The mouse models are indispensable for elucidation of the pathogenic mechanism and for development of therapeutic approaches, since they can be easily and reliably reproduced. In this section, we introduce the pathological features of $m d x$ and $m d x 52$ mice and the availability of therapeutic approaches which have benefited from the use of these mouse models.

\subsection{Mdx Mice}

4.1.1. Pathological Characteristics. X-linked muscular dystrophy mouse $(m d x)$ was found to spontaneously occur in a C57BL/10 strain [15], and a nonsense mutation was identified in exon 23 of the $D M D$ gene. This mutation causes a lack of dystrophin at the sarcolemma [2]. A previous study using $m d x$ mice revealed that muscle necrosis with 


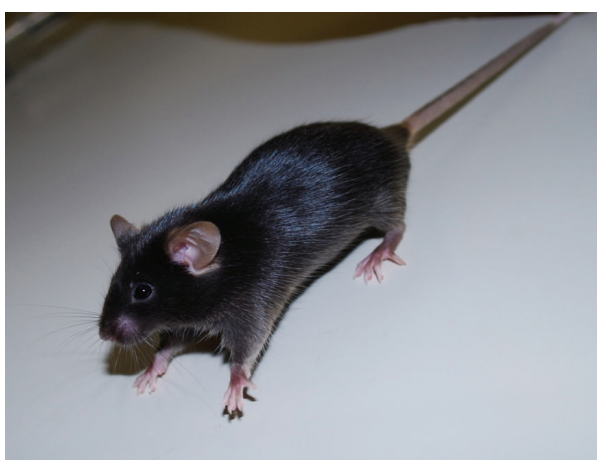

(a)

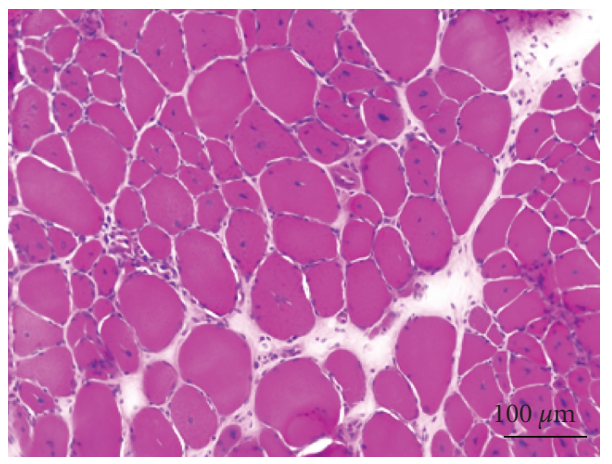

(b)

Figure 1: (a) A male $m d x 52$ mouse at 6 weeks of age. The generalized appearance is almost normal. (b) The pathology ( $\mathrm{H}$ and E) of the tibialis anterior muscle shows multicentrally nucleated fibers with an increased extent of invasion of inflammatory cells and the interstitial space. Hypertrophic muscle fibers were also observed.

infiltration of neutrophils or macrophages is recognized at approximately 2 weeks of age and that a massive muscle degeneration/necrosis occurs at approximately 1 month of age. Muscles continue to go through cycles of necrosis and regeneration throughout the lifespan of the $m d x$ mouse; it is just that is slows and is milder after 12 weeks. Necrotic fibers can be found at any age and appear with increasing frequency after 18 months of age and the pathology progresses. The muscle pathology is most pronounced between 2 and 8 weeks of age. This period is characterized by the presence of extensive necrosis, regenerated centrally nucleated fibers, and high levels of serum creatine kinase (CK), a biochemical marker of muscle necrosis $[11,15]$. The skeletal and cardiac muscle deterioration of the $m d x$ mouse is relatively much milder than that of human cases of DMD [16, 17]. As a result, the fibrosis and infiltration of inflammatory cells in the skeletal muscle at later stages tend to be much less than that observed in DMD patients.

4.1.2. Therapeutic Applications. Recent advances in therapeutic applications include exon skipping, gene therapy, and cell therapy. In the development of exon skipping therapy, the $m d x$ mouse is available for exon 23 skipping to convert an out-of-frame mutation into an in-frame mutation. Systemic administration of $2^{\prime}$-O-methyl phosphorothioate antisense oligonucleotides (2OMeAO) with a nonion polymer F127 to $m d x$ mice revealed that dystrophin is expressed in the whole-body skeletal muscle with the exception of heart muscle. However, the $2 \mathrm{OMeAO}$ did not reach a therapeutic level [18]. Meanwhile, systemic induction of dystrophin expression by phosphorodiamidate morpholino oligomers (PMO: morpholino) in the $m d x$ mouse reached a treatable level in whole-body skeletal muscle with the exception of the heart $[19,20]$. The peptide-linked PMO (PPMO) induced high expression of dystrophin not only in wholebody skeletal muscles but also in the heart in $m d x$ mice [21]. A unique exon-skipping method was proposed in which the mutated exon 23 on the mRNA of $m d x$ mice is removed by a single administration of an AAV vector expressing antisense sequences linked to a modified U7 small nuclear RNA [22].

In the development of gene therapy, we and other groups have designed a shorter but functional microdystrophin to incorporate into the recombinant AAV2 (rAAV2). The microdystrophin, which has a large deletion in the central rod domain, was constructed on the basis of the sequence of the mutated DMD gene in a BMD patient with a nearly normal life expectancy. The expression of this construct was driven by the muscle-specific creatine kinase (MCK) promoter, and local and systemic delivery of rAAV2 was found to restore the muscle function and life span of $m d x$ mice $[8,9]$. The other serotypes-rAAV6 [23], rAAV8 [24], and rAAV9 [25] - are also expected to be useful for systemic delivery of the DMD gene.

Although certain issues such as body size, genetic background, and pathological features should be addressed, the $m d x$ mouse has been indispensable as a DMD model for development of therapeutic approaches.

\subsection{Mdx52 Mouse}

4.2.1. Pathological Characteristics. Katsuki and colleagues successfully generated a new DMD mouse model known as $m d x 52$. In this model, exon 52 of the murine DMD gene was deleted using a homologous recombination technique [13]. Like the $m d x$ mouse, $m d x 52$ lacks dystrophin and presents dystrophic changes with muscle hypertrophy (Figure 1). In particular, the retina-specific dystrophin isoform Dp260 is absent and abnormalities were observed in electroretinographic analyses [26].

4.2.2. Therapeutic Applications. The targeting of exon 51 for exon skipping is theoretically applicable to the highest percentage (13\%) of DMD patients with an out-of frame deletion mutation [27-29]. An in-frame dystrophin mutation performed by an exon 51 skipping technique via local intramuscular injection of 2OMeAOs was found to be successful in some patients with DMD $[30,31]$. Recently, we conducted exon 51 skipping using PMO in $m d x 52$ mice to convert an out-of-frame mutation into an in-frame mutation and examined muscle function. The results showed that dystrophin expression was restored in whole-body skeletal muscles with amelioration of the dystrophic pathology and 
improved muscle function [32]. Thus, $m d x 52$ has proven to be useful for development of the exon skipping technique and was used to obtain proof of concept for ongoing clinical trials.

\section{Canine Model of DMD}

Several different canine models of DMD have been reported thus far [4]. Mutations of the canine DMD gene were identified in Golden Retriever [33], Rottweiler [34], German Short-Hair Pointer [35], and Cavalier King Charles Spaniels [36]. Golden Retriever muscular dystrophy (GRMD) has been the most extensively examined and characterized. We recently described Beagle-based canine X-linked muscular dystrophy $\left(\mathrm{CXMD}_{\mathrm{J}}\right)$ and conducted many studies on the elucidation of pathogenesis and the development of therapeutic approaches using this model. Exon-skipping therapy has been investigated using Cavalier King Charles Spaniels with muscular dystrophy (CKCS-MD). In this section, we describe the pathological characteristics and availability of gene therapies for GRMD, CXMD , and CKCS-MD.

\subsection{Golden Retriever Muscular Dystrophy (GRMD)}

5.1.1. Pathological Characteristics. GRMD is characterized by progressive skeletal muscle weakness and atrophy as well as cardiac involvement. These characteristics are similar to those of DMD. GRMD is caused by a point mutation at the intron 6 splice acceptor site of the canine DMD gene. This causes skipping of exon 7 and a premature stop codon in exon 8 and results in a lack of dystrophin [33]. The level of serum CK is dramatically increased early at 1-2 days of age and extensive diaphragm damage is observed [37]. The clinical manifestations of GRMD are progressive with the gradual loss of muscle mass and contractures that often lead to skeletal deformities [37]. Extensive muscle degeneration and necrosis of generalized muscles are identified from birth onwards [38]. A distinct feature is enlargement of the base of the tongue, pharyngeal muscle, and esophagus, resulting in dysphagia, drooling, and regurgitation. Severe fibrosis in muscle and joint contracture develops by 6 months of age [33], and respiratory failure or cardiomyopathy are frequently observed at younger ages [39]. GRMD is further characterized by progressive cardiomyopathy as indicated by deeper and narrower $\mathrm{Q}$ waves in leads II, III, and aVF relative to cardiac involvement in human DMD patients [38, 40,41].

The skeletal and cardiac characteristics of GRMD are more similar to those of DMD than of $m d x$. The genetic background and body size of the Golden Retriever is closer to human than the mouse. Therefore, GRMD has been considered to be a useful animal model for human DMD in recent years.

5.1.2. Therapeutic Applications. Preliminary gene therapy experiments on GRMD performed using adenovectors [42], and high expression of a dystrophin minigene was achieved in GRMD using replication-deficient adenoviral vectors [43].
Very recently, systemic delivery of the AAV9 human minidystrophin vector induced widespread muscle expression of the transgene in neonatal dystrophic dogs [44].

In exon-skipping therapy, prolonged maintenance of functional dystrophin in GRMD muscle has additionally been achieved through chimeric RNA/DNA oligonucleotide therapy [45]. GRMD muscle cells were observed in order to compare the effectiveness of $2 \mathrm{OMe} \mathrm{AO}, \mathrm{PMO}$, or peptidelinked PMO (PPMO) [46]. The PPMO was found to be capable of inducing high and sustained levels of exon skipping and induced the highest level of dystrophin expression with no apparent adverse effects upon the cells.

\subsection{Canine X-Linked Muscular Dystrophy $\left(C X M D_{J}\right)$}

5.2.1. Pathological Characteristics. As described in the previous section, GRMD is well characterized and attractive for research on DMD. However, Golden Retrievers are too large to be treated or raised easily and animal trials employing these dogs have substantial animal welfare implications and high costs associated with both maintenance and treatment. To address these issues, we developed a strain of mediumsized dystrophic Beagles by artificial insemination of two females with thawed spermatozoa obtained from a Golden Retriever with GRMD followed by interbreed crossing of the carrier female dogs with Beagle sires [47]. We designated the dystrophic dogs as canine X-linked muscular dystrophy in Japan $\left(\mathrm{CXMD}_{\mathrm{J}}\right)$. The colony is maintained at the General Animal Research Facility of the National Center of Neurology and Psychiatry in Tokyo [47]. The serum CK level of dogs with $\mathrm{CXMD}_{\mathrm{J}}$ at birth is very high, and the mortality rate during the neonatal period is about $32.3 \%$. This is significantly higher than that of normal littermates (13.3\%). Diaphragm muscle involvement occurs shortly after birth and is more severe than that of limb muscles. At an age of about 2-3 months, atrophy and weakness of limb muscles appear, followed by development of macroglossia, dysphasia, gait disturbance, and joint contracture from 4 months of age. These symptoms rapidly progress until about 10 months of age, after which the progression of the disease is retarded [48]. The clinical manifestations of third generation $\mathrm{CXMD}_{\mathrm{J}}$ are similar but milder than those observed in GRMD (Figure 2).

In the cardiac manifestation, GRMD as well as DMD have particular characteristics such as distinct deep electrocardiogram Q-waves and fibrosis of the left ventricular wall $[38,40,41]$. We regularly examined the cardiac performance of the $\mathrm{CXMD}_{\mathrm{J}}$ model by electrocardiogram, echocardiogram, and pathology and found that the deep Q-waves on echocardiograms precede the development of overt fibrosis in histopathology [49]. So far, the pathogenic mechanism of the distinct deep Q-waves has been considered to be ascribed to fibrosis in the posterobasal region of the left ventricular wall in DMD [41]. When we investigated the cardiac conduction system of $\mathrm{CXMD}_{\mathrm{J}}$, a remarkable extent of vacuolar degeneration was observed in Purkinje fibers despite the absence of detectable fibrotic lesions in the ventricular myocardium [50]. The degenerated Purkinje fibers 


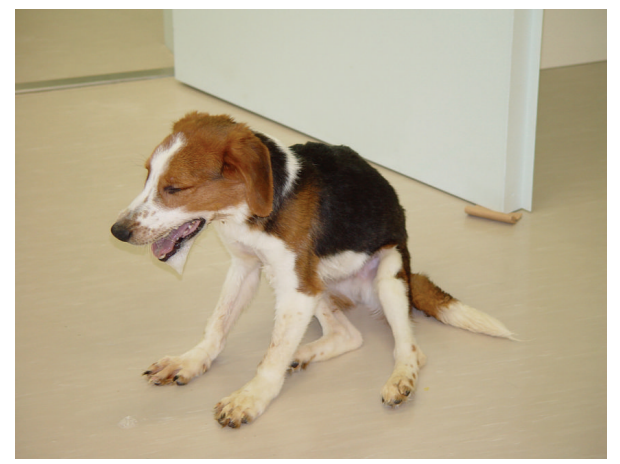

(a)

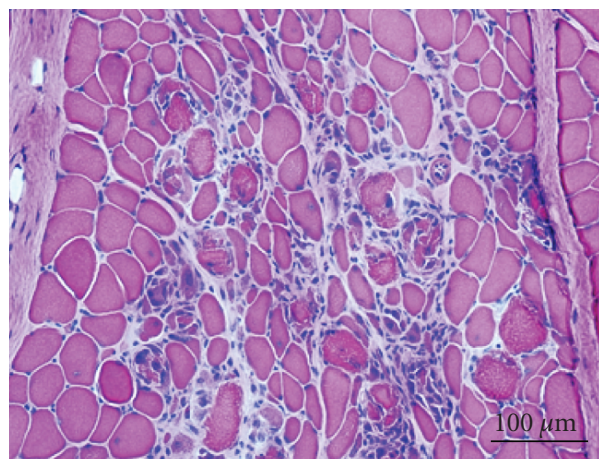

(b)

Figure 2: (a) A male canine with X-linked muscular dystrophy $\left(\mathrm{CXMD}_{\mathrm{J}}\right)$ at 6 months of age. The atrophy of muscles throughout the body (including temporal muscle) is observed. Kyphosis, abnormal sitting posture, and contracture of hindlimb joints are seen. (b) The pathology ( $\mathrm{H}$ and $\mathrm{E}$ ) of tibialis cranialis muscle indicates muscle necrosis with infiltration of inflammatory cells, centrally nucleated fibers, and increased interstitial connective tissues.

were coincident with overexpression of Dp71 (a C-terminal truncated isoform of dystrophin) at the sarcolemma and translocation of $\mu$-calpain (a calcium-dependent protease) to the periphery near the sarcolemma or to the vacuoles. Utrophin, a homologue of dystrophin was upregulated in dystrophin deficiency. Utrophin was found to be highly upregulated in the Purkinje fibers in the early stage, but the expression was dislocated when vacuolar degeneration was recognized at 4 months of age. Based on these findings, we hypothesized that the selective degeneration of Purkinje fibers could be associated with distinct deep Q-waves on electrocardiograms and the fatal arrhythmia which is observed in dystrophinopathy [50]. Thus, our previous investigations of the clinical phenotype in $\mathrm{CXMD}_{\mathrm{J}}$ revealed not only that the phenotypes are nearly identical to those of GRMD but also that the model will play a useful role in elucidation of the pathogenic mechanism and in development of therapeutic approaches.

5.2.2. Therapeutic Applications. Recently, in conjunction with our collaborators at the Children's National Medical Center in the USA, we developed a multiexon-skipping technique for targeting exons 6 and 8 to convert an out-of-frame mutation into an in-frame mutation using PMOs. Systemic multiexon-skipping treatment in CXMD was found to restore dystrophin in the whole-body skeletal muscle (with the exception of heart). Furthermore, skeletal muscle function was notably improved without any adverse effects [51]. This represents the first report on successful in vivo multiexon-skipping. Furthermore, we have succeeded in producing multiexon skipping by administration of the PMOs used in CXMD to MyoD-transduced fibroblasts from a DMD patient with the exon 7 deletion of the $D M D$ gene [52]. This suggests that multiexon skipping is feasible for treatment of DMD patients and that this approach could be applicable to up to $83 \%$ of all DMD patients [27].

To examine therapeutic effects and safety in a larger animal model, we have examined the efficiency of rAAV2 infection of canine myotubes and expression of the lac $Z$ gene into normal canine muscle. In contrast to the results of $m d x$ investigations, the rAAV2-mediated gene transfer was found to elicit a severe immune response against the AAV particle, and its gene product in the host [53]. We, therefore, have examined the transgene expression and host immune response to two different rAAV (rAAV2 and rAAV8) in normal canine and in the CXMD J model after intramuscular injection and systemic administration by limb perfusion to bypass the immune activation of dendritic cells in the injected muscle. In contrast to the results with rAAV2 transduction, intramuscular transduction of rAAV8lac $Z$ in normal dogs and systemic administration of rAAV8microdystrophin in $\mathrm{CXMD}_{\mathrm{J}}$ were found to improve the expression of both transgenes in the skeletal muscle [54]. The $\mathrm{CXMD}_{\mathrm{J}}$ model led us to propose that rAAV8-mediated transduction strategy could have a therapeutic advantage in $D M D$ gene therapy.

Thus, the clinical phenotype of $\mathrm{CXMD}_{\mathrm{J}}$ has been well characterized as an appropriate animal model and the similarity of pathology of DMD is regarded as the most appropriate model for DMD in clinical trials. Other therapeutic approaches will be evaluated in the dog model with a view to establishing feasible protocols.

\subsection{Cavalier King Charles Spaniels with Muscular Dystrophy (CKCS-MD)}

5.3.1. Pathological Characteristics. Very recently, it was reported that another dystrophic dog, the Cavalier King Charles Spaniel with dystrophin-deficient muscular dystrophy (CKCS-MD) has a severe phenotype. This canine model has a missense mutation in the $5^{\prime}$ donor splice site of exon 50 resulting in deletion of exon 50 in mRNA transcripts and a predicted premature truncation of the translated protein [36].

5.3.2. Therapeutic Applications. The therapeutic strategy of exon skipping in the GRMD or CXMD J models provides a significant advantage in clinical trials, but also has the unfavorable characteristic that the disease-causing mutation does not include the region of the DMD gene that is most commonly mutated in human DMD patients. About $60 \%$ of 
DMD patients harbor deletions in exons 45-55 of the DMD gene $[55,56]$. As described previously, the exon 51 skipping technique, which is being employed in clinical trials $[30,31]$, is applicable to DMD patients which have deletions of exon 50 , exon 52, exons 48-50, or exons 49-50, among others, and is feasible for more patients than other exon skipping strategies $[27,57,58]$. AO-mediated skipping of exon 51 in cultured myoblasts of the CKCS-MD dog restored the reading frame and protein expression [17]. This observation suggests that the use of this canine model would be valuable in the preclinical trials of therapy based upon exon 51 skipping.

\section{Feline Model of DMD}

6.1. Hypertrophy Feline Muscular Dystrophy (HFMD). Feline muscular dystrophy with dystrophin deficiency has been identified [59]. This feline model has a unique phenotypic expression of hypertrophy of the tongue, neck, and shoulder muscles, lingual calcification, excessive salivation, megaesophagus, gait disturbance manifesting as bunny hopping, dilated cardiomyopathy, hepatosplenomegaly and kidney failure $[60,61]$. These dystrophic cats have been described as having hypertrophic feline muscular dystrophy (HFMD) because of the distinct hypertrophic feature. The pathology exhibits muscle degeneration and centrally nucleated regeneration and accumulation of calcium deposits within muscle fibers without development of fibrosis. The HFMD cat has a large deletion of muscle and Purkinje promoters resulting in a lack of dystrophin in the skeletal and cardiac muscle [62]. However, the dystrophic cats have not been widely used as a DMD model due to the limited pathological similarity to DMD [4]; there have been no reports on therapeutic approaches using this feline model.

\section{Conclusions}

Murine models will continue to provide important findings for the basic study of pathogenesis and development of therapies, but the clinical phenotype is mild and this is a weak point. On the other hand, canine models have severe skeletal and cardiac defects resembling DMD and the body size and genetic background of canines are more similar to human beings than the murine models. We are, therefore, convinced that canine models will be more useful for future contributions to preclinical study of newly developed therapies.

\section{Acknowledgment}

These studies were supported by Health Sciences Research Grants for Research on Psychiatric and Neurological Diseases and Mental Health (nos. H12-kokoro-025, H15-kokoro021, and H18-kokoro-019), the Human Genome and Gene Therapy (nos. H13-genome-001, and H16-genome-003), and the Health and Labor Sciences Research Grants for Translation Research (no. H19-translational research-003) from the Ministry of Health, Labor and Welfare of Japan, and Grants-in-Aid for Scientific Research from the Ministry of Education, Science, Sports and Culture of Japan (no. 21300157 to A. Nakamura).

\section{References}

[1] H. Moser, "Duchenne muscular dystrophy: pathogenetic aspects and genetic prevention," Human Genetics, vol. 66, no. 1, pp. 17-40, 1984.

[2] E. P. Hoffman, R. H. Brown Jr., and L. M. Kunkel, "Dystrophin: the protein product of the Duchenne muscular dystrophy locus," Cell, vol. 51, no. 6, pp. 919-928, 1987.

[3] K. P. Campbell, "Three muscular dystrophies: loss of cytoskeleton-extracellular matrix linkage," Cell, vol. 80, no. 5, pp. 675-679, 1995.

[4] G. D. Shelton and E. Engvall, "Canine and feline models of human inherited muscle diseases," Neuromuscular Disorders, vol. 15, no. 2, pp. 127-138, 2005.

[5] E. W. Yeung, N. P. Whitehead, T. M. Suchyna, P. A. Gottlieb, F. Sachs, and D. G. Allen, "Effects of stretch-activated channel blockers on $\left[\mathrm{Ca}^{2+}\right]_{\mathrm{i}}$ and muscle damage in the mdx mouse," Journal of Physiology, vol. 562, no. 2, pp. 367-380, 2005.

[6] M. Koening, A. H. Beggs, M. Moyer et al., "The molecular basis for Duchenne versus Becker muscular dystrophy: correlation of severity with type of deletion," American Journal of Human Genetics, vol. 45, no. 4, pp. 498-506, 1989.

[7] C. Wilson and A. D. Keefe, "Building oligonucleotide therapeutics using non-natural chemistries," Current Opinion in Chemical Biology, vol. 10, no. 6, pp. 607-614, 2006.

[8] S. Karkare and D. Bhatnagar, "Promising nucleic acid analogs and mimics: characteristic features and applications of PNA, LNA, and morpholino," Applied Microbiology and Biotechnology, vol. 71, no. 5, pp. 575-586, 2006.

[9] M. Yoshimura, M. Sakamoto, M. Ikemoto et al., "AAV vectormediated microdystrophin expression in a relatively small percentage of mdx myofibers improved the mdx phenotype," Molecular Therapy, vol. 10, no. 5, pp. 821-828, 2004.

[10] P. Gregorevic, J. M. Allen, E. Minami et al., "rAAV6microdystrophin preserves muscle function and extends lifespan in severely dystrophic mice," Nature Medicine, vol. 12, no. 7, pp. 787-789, 2006.

[11] G. R. Coulton, N. A. Curtin, J. E. Morgan, and T. A. Partridge, "The mdx mouse skeletal muscle myopathy: II. Contractile properties," Neuropathology and Applied Neurobiology, vol. 14, no. 4, pp. 299-314, 1988.

[12] W. B. Im, S. F. Phelps, E. H. Copen, E. G. Adams, J. L. Slightom, and J. S. Chamberlain, "Differential expression of dystrophin isoforms in strains of mdx mice with different mutations," Human Molecular Genetics, vol. 5, no. 8, pp. 1149-1153, 1996.

[13] E. Araki, K. Nakamura, K. Nakao et al., "Targeted disruption of exon 52 in the mouse dystrophin gene induced muscle degeneration similar to that observed in duchenne muscular dystrophy," Biochemical and Biophysical Research Communications, vol. 238, no. 2, pp. 492-497, 1997.

[14] A. E. Deconinck, J. A. Rafael, J. A. Skinner et al., "Utrophindystrophin-deficient mice as a model for Duchenne muscular dystrophy," Cell, vol. 90, no. 4, pp. 717-727, 1997.

[15] G. Bulfield, W. G. Siller, P. A. L. Wight, and K. J. Moore, "X chromosome-linked muscular dystrophy ( $\mathrm{mdx}$ ) in the mouse," Proceedings of the National Academy of Sciences of the United States of America, vol. 81, no. 4 I, pp. 1189-1192, 1984.

[16] J. Dangain and G. Vrbova, "Muscle development in $\mathrm{mdx}$ mutant mice," Muscle and Nerve, vol. 7, no. 9, pp. 700-704, 1984. 
[17] Y. Tanabe, K. Esaki, and T. Nomura, "Skeletal muscle pathology in X chromosome-linked muscular dystrophy ( $\mathrm{mdx}$ ) mouse," Acta Neuropathologica, vol. 69, no. 1-2, pp. 91-95, 1986.

[18] Q. L. Lu, C. J. Mann, F. Lou et al., "Functional amounts of dystrophin produced by skipping the mutated exon in the $\mathrm{mdx}$ dystrophic mouse," Nature Medicine, vol. 9, no. 8, pp. 10091014, 2003.

[19] D. J. Wells, "Therapeutic restoration of dystrophin expression in Duchenne muscular dystrophy," Journal of Muscle Research and Cell Motility, vol. 27, no. 5-7, pp. 387-398, 2006.

[20] S. Fletcher, K. Honeyman, A. M. Fall, P. L. Harding, R. D. Johnsen, and S. D. Wilton, "Dystrophin expression in the mdx mouse after localised and systemic administration of a morpholino antisense oligunucleotide," Journal of Gene Medicine, vol. 8, no. 2, pp. 207-216, 2006.

[21] N. Jearawiriyapaisarn, H. M. Moulton, B. Buckley et al., "Sustained dystrophin expression induced by peptide-conjugated morpholino oligomers in the muscles of mdx mice," Molecular Therapy, vol. 16, no. 9, pp. 1624-1629, 2008.

[22] A. Goyenvalle, A. Vulin, F. Fougerousse et al., "Rescue of dystrophic muscle through U7 snRNA-mediated exon skipping," Science, vol. 306, no. 5702, pp. 1796-1799, 2004.

[23] P. Gregorevic, M. J. Blankinship, J. M. Allen et al., "Systemic delivery of genes to striated muscles using adeno-associated viral vectors," Nature Medicine, vol. 10, no. 8, pp. 828-834, 2004.

[24] Z. Wang, T. Zhu, C. Qiao et al., "Adeno-associated virus serotype 8 efficiently delivers genes to muscle and heart," Nature Biotechnology, vol. 23, no. 3, pp. 321-328, 2005.

[25] L. T. Bish, K. Morine, M. M. Sleeper et al., "Adeno-associated virus (AAV) serotype 9 provides global cardiac gene transfer superior to AAV1, AAV6, AAV7, and AAV8 in the mouse and rat," Human Gene Therapy, vol. 19, no. 12, pp. 1359-1368, 2008.

[26] S. Kameya, E. Araki, M. Katsuki et al., "Dp260 disrupted mice revealed prolonged implicit time of the b-wave in ERG and loss of accumulation of $\beta$-dystroglycan in the outer plexiform layer of the retina," Human Molecular Genetics, vol. 6, no. 13, pp. 2195-2203, 1997.

[27] A. Aartsma-Rus, I. Fokkema, J. Verschuuren et al., "Theoretic applicability of antisense-mediated exon skipping for Duchenne muscular dystrophy mutations," Human Mutation, vol. 30, no. 3, pp. 293-299, 2009.

[28] M. Kinali, V. Arechavala-Gomeza, L. Feng et al., "Local restoration of dystrophin expression with the morpholino oligomer AVI-4658 in Duchenne muscular dystrophy: a single-blind, placebo-controlled, dose-escalation, proof-ofconcept study," The Lancet Neurology, vol. 8, no. 10, pp. 918928, 2009.

[29] A. T. J. M. Helderman-van den Enden, C. S. M. Straathof, A. Aartsma-Rus et al., "Becker muscular dystrophy patients with deletions around exon 51; a promising outlook for exon skipping therapy in Duchenne patients," Neuromuscular Disorders, vol. 20, no. 4, pp. 251-254, 2010.

[30] J. C. van Deutekom, A. A. Janson, I. B. Ginjaar et al., "Local dystrophin restoration with antisense oligonucleotide PRO051," The New England Journal of Medicine, vol. 357, no. 26, pp. 2677-2686, 2007.

[31] F. Muntoni, K. Bushby, and G. Van Ommen, "128th ENMC International Workshop on 'Preclinical optimization and phase I/II clinical trials using antisense oligonucleotides in Duchenne muscular dystrophy' 22-24 October 2004, Naarden,
The Netherlands," Neuromuscular Disorders, vol. 15, no. 6, pp. 450-457, 2005.

[32] Y. Aoki, A. Nakamura, T. Yokota et al., "In-frame dystrophin following exon 51-skipping improves muscle pathology and function in the exon 52-deficient $m d x$ mouse," Molecular Therapy, vol. 18, no. 11, pp. 1995-2005, 2010.

[33] N. J. H. Sharp, J. N. Kornegay, S. D. van Camp et al., "An error in dystrophin mRNA processing in golden retriever muscular dystrophy, an animal homologue of Duchenne muscular dystrophy," Genomics, vol. 13, no. 1, pp. 115-121, 1992.

[34] N. Winand, D. Pradham, and B. Cooper, "Molecular characterization of severe Duchenne-type muscular dystrophy in a family of Rottwiler dogs," in Molecular Mechanism of Neuromuscular Disease, Muscular Dystrophy Association, Tucson, Ariz, USA, 1994.

[35] S. J. Schatzberg, N. J. Olby, M. Breen et al., "Molecular analysis of a spontaneous dystrophin 'knockout' dog," Neuromuscular Disorders, vol. 9, no. 5, pp. 289-295, 1999.

[36] G. L. Walmsley, V. Arechavala-Gomeza, M. Fernandez-Fuente et al., "A duchenne muscular dystrophy gene hot spot mutation in dystrophin-deficient cavalier king charles spaniels is amenable to exon 51 skipping," PloS one, vol. 5, no. 1, Article ID e8647, 2010.

[37] B. J. Cooper, N. J. Winand, H. Stedman et al., "The homologue of the Duchenne locus is defective in X-linked muscular dystrophy of dogs," Nature, vol. 334, no. 6178, pp. 154-156, 1988.

[38] B. A. Valentine, B. J. Cooper, A. De Lahunta, R. O'Quinn, and J. T. Blue, "Canine X-linked muscular dystrophy. An animal model of Duchenne muscular dystrophy: clinical studies," Journal of the Neurological Sciences, vol. 88, no. 1-3, pp. 69-81, 1988.

[39] F. Nguyen, Y. Cherel, L. Guigand, I. Goubault-Leroux, and M. Wyers, "Muscle lesions associated with dystrophin deficiency in neonatal golden retriever puppies," Journal of Comparative Pathology, vol. 126, no. 2-3, pp. 100-108, 2002.

[40] N. S. Moise, B. A. Valentine, C. A. Brown et al., "Duchenne's cardiomyopathy in a canine model: electrocardiographic and echocardiographic studies," Journal of the American College of Cardiology, vol. 17, no. 3, pp. 812-820, 1991.

[41] J. K. Perloff, W. C. Roberts, A. C. de Leon Jr., and D. O'Doherty, "The distinctive electrocardiogram of Duchenne's progressive muscular dystrophy. An electrocardiographicpathologic correlative study," The American Journal of Medicine, vol. 42, no. 2, pp. 179-188, 1967.

[42] J. M. Howell, S. Fletcher, B. A. Kakulas, M. O’Hara, H. Lochmuller, and G. Karpati, "Use of the dog model for Duchenne muscular dystrophy in gene therapy trials," Neuromuscular Disorders, vol. 7, no. 5, pp. 325-328, 1997.

[43] J. M. Howell, H. Lochmüller, A. O’Hara et al., "High-level dystrophin expression after adenovirus-mediated dystrophin minigene transfer to skeletal muscle of dystrophic dogs: prolongation of expression with immunosuppression," Human Gene Therapy, vol. 9, no. 5, pp. 629-634, 1998.

[44] J. N. Kornegay, J. Li, J. R. Bogan et al., "Widespread muscle expression of an AAV9 human mini-dystrophin vector after intravenous injection in neonatal dystrophin-deficient dogs," Molecular Therapy, vol. 19, no. 8, pp. 1501-1508, 2010.

[45] R. J. Bartlett, S. Stockinger, M. M. Denis et al., "In vivo targeted repair of a point mutation in the canine dystrophin gene by a chimeric RNA/DNA oligonucleotide," Nature Biotechnology, vol. 18, no. 6, pp. 615-622, 2000. 
[46] G. McClorey, H. M. Moulton, P. L. Iversen, S. Fletcher, and S. D. Wilton, "Antisense oligonucleotide-induced exon skipping restores dystrophin expression in vitro in a canine model of DMD," Gene Therapy, vol. 13, no. 19, pp. 1373-1381, 2006.

[47] Y. Shimatsu, K. Katagiri, T. Furuta et al., "Canine X-linked muscular dystrophy in Japan (CXMD)," Experimental Animals, vol. 52, no. 2, pp. 93-97, 2003.

[48] Y. Shimatsu, M. Yoshimura, K. Yuasa et al., "Major clinical and histopathological characteristics of canine X-linked muscular dystrophy in Japan, CXMD," Acta Myologica, vol. 24, no. 2, pp. 145-154, 2005.

[49] N. Yugeta, N. Urasawa, Y. Fujii et al., "Cardiac involvement in Beagle-based canine X-linked muscular dystrophy in Japan (CXMD): electrocardiographic, echocardiographic, and morphologic studies," BMC Cardiovascular Disorders, vol. 6, article 47, 2006.

[50] N. Urasawa, M. R. Wada, N. Machida et al., "Selective vacuolar degeneration in dystrophin-deficient canine Purkinje fibers despite preservation of dystrophin-associated proteins with overexpression of Dp71," Circulation, vol. 117, no. 19, pp. 2437-2448, 2008.

[51] T. Yokota, Q. L. Lu, T. Partridge et al., "Efficacy of systemic morpholino exon-skipping in duchenne dystrophy dogs," Annals of Neurology, vol. 65, no. 6, pp. 667-676, 2009.

[52] T. Saito, A. Nakamura, Y. Aoki et al., "Antisense PMO found in dystrophic dog model was effective in cells from exon 7deleted DMD patient," PLoS One, vol. 5, no. 8, Article ID e12239, 2010.

[53] K. Yuasa, M. Yoshimura, N. Urasawa et al., "Injection of a recombinant AAV serotype 2 into canine skeletal muscles evokes strong immune responses against transgene products," Gene Therapy, vol. 14, no. 17, pp. 1249-1260, 2007.

[54] S. Ohshima, J. H. Shin, K. Yuasa et al., "Transduction efficiency and immune response associated with the administration of AAV8 vector into dog skeletal muscle," Molecular Therapy, vol. 17, no. 1, pp. 73-80, 2009.

[55] J. Alter, F. Lou, A. Rabinowitz et al., "Systemic delivery of morpholino oligonucleotide restores dystrophin expression bodywide and improves dystrophic pathology," Nature Medicine, vol. 12, no. 2, pp. 175-177, 2006.

[56] A. Aartsma-Rus, A. A. M. Janson, W. E. Kaman et al., "Antisense-induced multiexon skipping for Duchenne muscular dystrophy makes more sense," American Journal of Human Genetics, vol. 74, no. 1, pp. 83-92, 2004.

[57] V. Arechavala-Gomeza, I. R. Graham, L. J. Popplewell et al., "Comparative analysis of antisense oligonucleotide sequences for targeted skipping of exon 51 during dystrophin pre-mRNA splicing in human muscle," Human Gene Therapy, vol. 18, no. 9, pp. 798-810, 2007.

[58] A. Nakamura and S. Takeda, "Exon-skipping therapy for Duchenne muscular dystrophy," Neuropathology, vol. 29, no. 4, pp. 494-501, 2009.

[59] J. H. Vos, J. S. van der Linde-Sipman, and S. A. Goedegebuure, "Dystrophy-like myopathy in the cat," Journal of Comparative Pathology, vol. 96, no. 3, pp. 335-341, 1986.

[60] J. L. Carpenter, E. P. Hoffmann, F. C. A. Romanul et al., "Feline muscular dystrophy with dystrophin deficiency," The American Journal of Pathology, vol. 135, no. 5, pp. 909-919, 1989.

[61] F. P. Gaschen, E. P. Hoffman, J. R. M. Gorospe et al., "Dystrophin deficiency causes lethal muscle hypertrophy in cats," Journal of the Neurological Sciences, vol. 110, no. 1-2, pp. 149-159, 1992.
[62] N. J. Winand, M. Edwards, D. Pradhan, C. A. Berian, and B. J. Cooper, "Deletion of the dystrophin muscle promoter in feline muscular dystrophy," Neuromuscular Disorders, vol. 4, no. 5-6, pp. 433-445, 1994. 


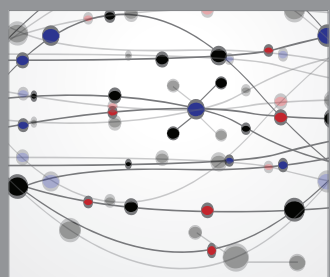

The Scientific World Journal
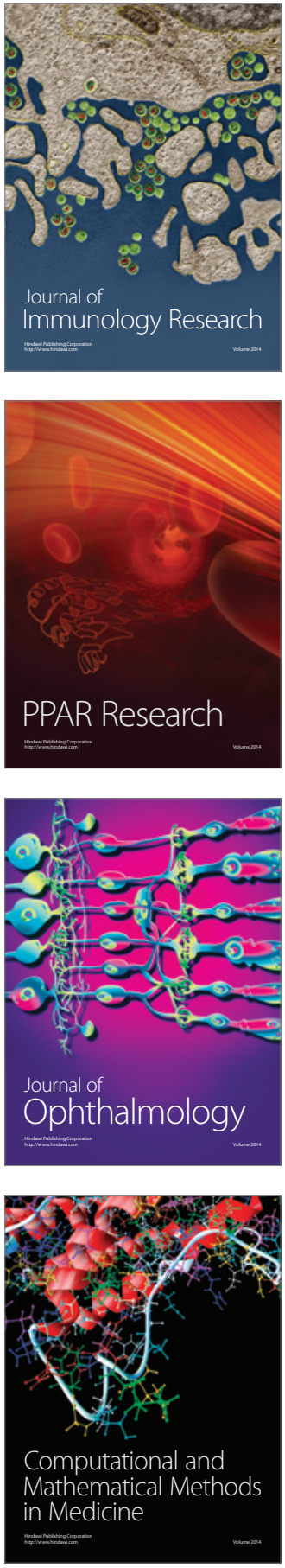

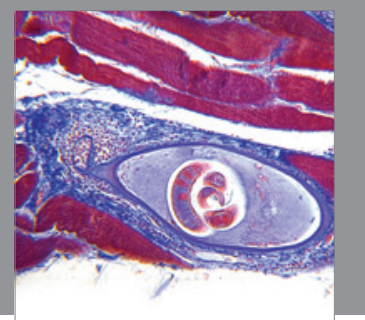

Gastroenterology

Research and Practice
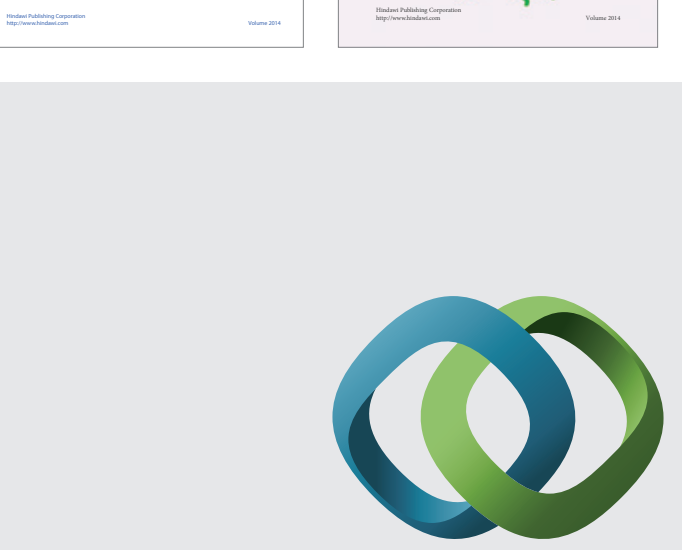

\section{Hindawi}

Submit your manuscripts at

http://www.hindawi.com
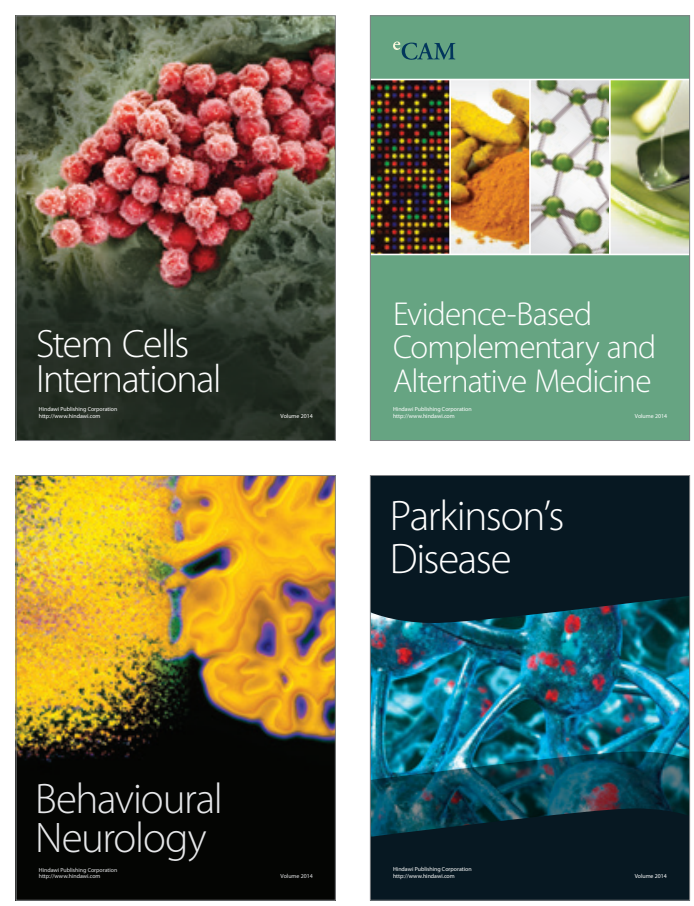

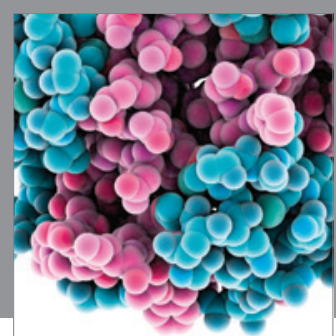

Journal of
Diabetes Research

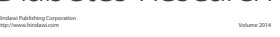

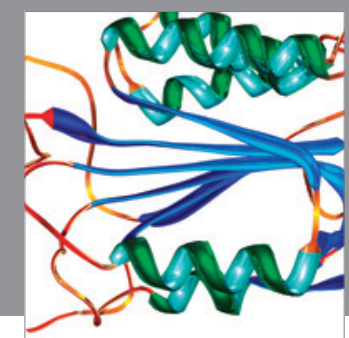

Disease Markers
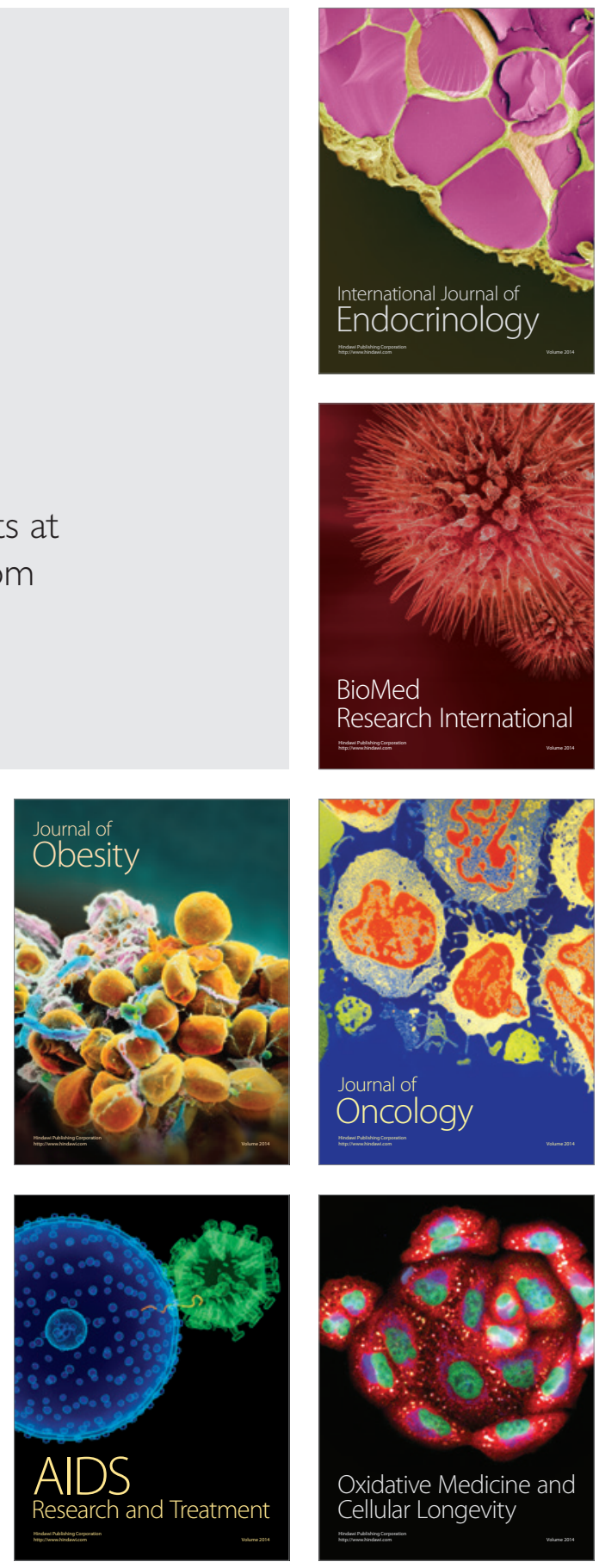